\title{
Received Signal Strength Quantization for Secure Indoor Positioning via Fingerprinting
}

\section{Richter, Philipp}

IEEE

2018

Richter , P , Yang , Z , Tkachenko , O , Leppäkoski , H , Järvinen , K, Schneider , T \& Lohan , E S 2018, Received Signal Strength Quantization for Secure Indoor Positioning via Fingerprinting . in 2018 8th International Conference on Localization and GNSS (ICL-GNSS) . International Conference on Localization and GNSS, IEEE, Piscataway, NJ , International Conference on Localization and GNSS (ICL-GNSS) , Guimaraes , Portugal , 31/03/2018 . https://doi.org/10.1109/ICL

http://hdl.handle.net/10138/308156

https://doi.org/10.1109/ICL-GNSS.2018.8440910

acceptedVersion

Downloaded from Helda, University of Helsinki institutional repository.

This is an electronic reprint of the original article.

This reprint may differ from the original in pagination and typographic detail.

Please cite the original version. 


\title{
Received Signal Strength quantization for secure indoor positioning via fingerprinting
}

\author{
P. Richter*, Z. Yang ${ }^{\dagger}$, O. Tkachenko, ${ }^{\ddagger}$, H. Leppäkoski*, K. Järvinen ${ }^{\dagger}$, T. Schneider, ${ }^{\ddagger}$ and E. S. Lohan* \\ *Tampere University of Technology, Tampere, Finland \\ ${ }^{\dagger}$ University of Helsinki, Helsinki, Finland \\ $\ddagger$ TU Darmstadt, Darmstadt, Germany \\ Email: philipp.richter@tut.fi
}

\begin{abstract}
The increasingly connected world magnifies the threats to users' location privacy. Encryption protocols offer solutions to privacy concerns, but they are computationally very demanding. A reduction of the bit-length of the Received Signal Strength (RSS) measurements is required for a realistic, privacy-preserving positioning system based on fingerprinting. This paper studies the practical design of quantizers for RSS fingerprinting data and analyses the effect of the quantization on the positioning performance, with several real data sets and positioning algorithms. Our results show that 4-bit quantization deteriorate the accuracy compared to no-quantization case by only $20 \mathrm{~cm}$ and that 1-bit approaches (i.e. proximity based positioning) are also feasible for certain applications.
\end{abstract}

Index Terms-Received Signal Strength (RSS), quantization, secure protocols, privacy-preserving positioning, fingerprinting

\section{INTRODUCTION AND MOTIVATION}

The Received Signal Strength (RSS) information in a wireless system is nowadays used in a variety of applications, ranging from link-budget computations and optimizations of the communication chain to RSS-based localization and tracking. Typically, the RSS are used without any quantization, but a quantized RSS approach would bring in significant benefits in terms of lowering the energy consumption and communication bandwidths [1] and increasing the security of the positioning protocol for RSS-based positioning. In this paper we address the latter case, namely the quantization of RSS values for the purpose of enabling low-complexity security protocols in positioning for an increased user privacy. Indeed, in a RSSbased positioning approach, there are two main threats to user's location privacy if the RSSs heard by the user are sent in "clear" (i.e. without any encryption mechanism):

- The location server in charge with computing the user's location can also track the users' position and could disclose it unwittingly to third parties.

- An attacker with the access to a fingerprint database of a particular building can intercept the user's signalling towards the location server (Medium Access Control (MAC) addresses of the Access Nodes (AN) and their corresponding RSS) and infer the user's location information.

User's position privacy infringements can bring in significant threats, as outlined recently in [2]. In order to offer solutions to the user's privacy problem, privacy-preserving protocols have to be derived. Security against external adversaries intercepting signals between the user and server is relatively easy to solve by encrypting the channel, e.g., with TLS. Privacy problems originating from the server's ability to track users is significantly harder to solve, but a few attempts are available in the literature [3]-[8]. Many of them use secure multiparty computation (MPC) based on partially homomorphic encryption that allows limited operations with encrypted data. Unfortunately, weaknesses have been identified recently in some of them [8]. The schemes also introduce significant computation and communication overheads compared to basic privacy-violating protocols. Nevertheless, some promising schemes have been identified in [8] based on MPC built from garbled circuits and additively homomorphic encryption. Their complexities are directly related to the precision (bits) of RSS values used in the protocols. Consequently, significant efficiency improvements could be received by using fewer bits in the quantized RSS values, both used in the fingerprint database and measured by the user's device.

While RSS quantization decreases the complexity of the privacy-preserving protocol, it will also decrease the accuracy of the location estimate. The goal of this paper is to investigate the impact of RSS quantization on the positioning accuracy.

Most related work about RSS quantization can be found in the research on sensor node localization in densely deployed wireless sensor networks, where sensors are low-cost with limited energy, communication and sensing ability. The authors of, for example, [9]-[11] use quantized RSS to localize a target in a sensor network, but they are not concerned about the trade-off between quantization and positioning accuracy.

The authors of [12] propose a quantizer whose output level is a function of the number of spatial grid cells, to minimize number of beacons while pertaining the positioning accuracy. The basic path loss model with log-normal shadowing is used in simulations to evaluate the method.

The studies [13] and [14] derive the Cramér-Rao lower bound (CRLB) to analyse the performance of a RSS-based localization system with quantized RSS. The principal objective in these studies are the optimal quantization thresholds based on the CRLB. Both works conclude that a small number of quantization levels suffices to achieve a good localization performance. [13] states that eight levels (3 bit) achieve a performance comparable with that of systems using nonquantized RSS. These contributions were later extended by [1] to a distributed estimation of the target location. They found that 
5-bit quantized RSS achieve a similar CRLB as using raw RSS.

These theoretical studies derive the optimal quantization thresholds for specific networks, network configurations and particular assumptions (a-priori knowledge of the sensor locations, isotropic signal attenuation model, reception of target's signals at all nodes, access nodes communicate with each other). This limits the validity of their findings and makes the transfer of the outcomes uncertain for positioning systems that do not reflect these assumption.

In [15] a genetic algorithm is used to find the partitions of a RSS quantizer. From experiments with EMSPCC 11 nodes in an $8 \times 12 \mathrm{~m}^{2}$ environment, they conclude that a 2-bit representation of RSS yields an adequate compromise between data compression and positioning accuracy.

Our study outlines privacy-preserving fingerprinting localization in WLANs and investigates the trade-off between positioning accuracy and quantization bit-length using realfield measurements in large multi-floor spaces (areas larger than $100 \times 100 \mathrm{~m}^{2}$ per floor). We design here several practical quantizers derived empirically from the fingerprint database and we evaluate them with $k$-Nearest Neighbour $(k$-NN) algorithms using three different distance metrics for five different WLAN RSS data sets in office and mall buildings.

\section{INDOOR POSITIONING WITH PRIVACY CONSTRAINTS}

Indoor positioning methods rely typically on inertial measurements or on radio signals, as those used in $\mathrm{WiFi}$ and Bluetooth [16]-[18]. Among the possible positioning techniques, fingerprinting with RSS measurements has been widely adopted, because the alternative techniques based on signal propagation times suffer severely from shadowing and multipath propagation effects and yield poorer accuracy. Its widespread use comes also from the ease with that the necessary data can be acquired and from its low complexity. With fingerprinting localization, a positioning accuracy of a few meters can be achieved, which is sufficient for many location based services.

RSS-based fingerprinting uses a pattern matching technique that finds the user's position by comparing an observed RSS signature, a set of RSS values from all ANs in range, with previously collected RSS signatures in a database. During an off-line phase, RSSs and the positions at which the RSSs have been recorded, are collected and stored in a database. In an on-line phase a RSS signature is measured and compared with the RSS signatures in the database. The position of the RSSs that match best with the observed RSSs serves as an estimate for the user position. For that comparison we use the $k$-NN method with the following commonly used metrics:

a) Gaussian-kernel distance:

$$
d_{i}=\sum_{m=1}^{M} \frac{1}{\sqrt{2 \pi \sigma_{s}^{2}}} \exp \left(-\frac{\left(s_{m}-\hat{s}_{m, i}\right)^{2}}{2 \sigma_{s}^{2}}\right)
$$

b) Euclidean distance:

$$
d_{i}=\sqrt{\sum_{m=1}^{M}\left(s_{m}-\hat{s}_{m, i}\right)^{2}}
$$

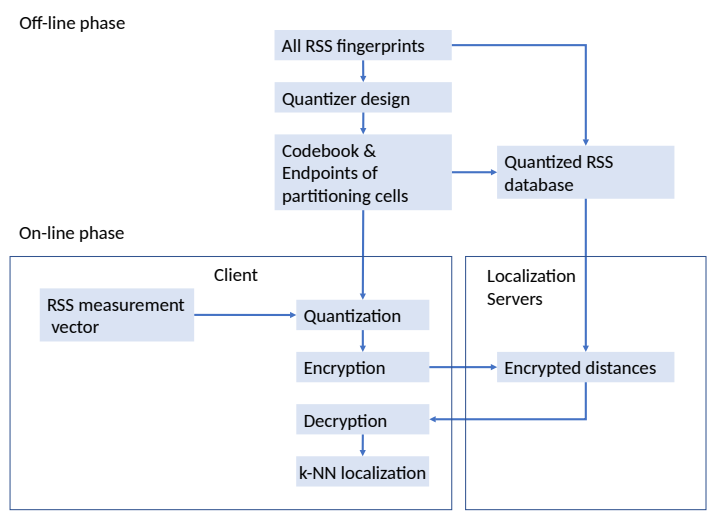

Fig. 1. Privacy preserving fingerprinting localization using quantized RSS.

c) Sørensen distance:

$$
d_{i}=\frac{\sum_{m=1}^{M}\left|s_{m}-\hat{s}_{m, i}\right|}{\sum_{m=1}^{N}\left(s_{m}+\hat{s}_{m, i}\right)},
$$

where $\left\{s_{m}\right\}_{m=1}^{M}$ is the observed RSS signature, $\left.\left\{\hat{s}_{m, i}\right\}\right)_{m=1}^{M}$ the $i$ th entry in the database and $M$ denotes the number of ANs.

To preserve the users' location privacy its RSS measurements need to be protected. Fig. 1 shows a flow chart of the privacypreserving positioning system that integrates quantization and encryption. In Sect. III, we describe possible privacy-preserving protocols and Sect. IV explains the quantization.

\section{SECURITY PROTOCOLS FOR POSITIONING}

In privacy-preserving RSS-based localization the problems are twofold: (1) how to prevent the server from learning the user's RSS measurements and, consequently, the user's location and (2) how to prevent the user from obtaining the server's database. Secure multi-party computation (MPC) are cryptographic protocols that allow two (or more) parties to jointly perform computations without revealing their inputs to each other. Yang and Järvinen [8] surveyed different possibilities to use MPC for efficient privacy-preserving RSSbased localization. They identified garbled circuits and additively homomorphic (Paillier) encryption as the main enabling techniques. In the following, we discuss the benefits of reducing the number of bits per RSS value in both of them.

\section{A. Garbled Circuits}

Garbled circuits introduced by Andrew Yao [19] allow two parties to jointly evaluate a function $f(x, y)$ without revealing their inputs ( $x$ and $y$, respectively) to each other. The simplest way to use this for privacy-preserving RSS-based localization is to let $x$ be the user's RSS measurements and $y$ be the server's database, but other more efficient ways have been proposed [8].

In MPC using garbled circuits, the main problem is the size of the garbled circuits that is proportional to the communication between the parties. The function $f$ is first represented as a Boolean circuit and then this circuit is scrambled into a garbled circuit so that each non-XOR gate in the circuit becomes a $2 \lambda$-bit table [20], where $\lambda=128$ is a typical value, and XORs 
are for free [21]. For instance, an addition (subtraction) of two $b$-bit integers requires a $2 b \lambda$-bit garbled circuit whereas a schoolbook multiplication requires a $2\left(2 b^{2}-b\right) \lambda$-bit garbled circuit (see, e.g., [22]). Given this, it is clear that the size of the garbled circuit for computing, for example, Eq. (2) depends heavily on the precision of RSS values.

Example: Consider constructing a garbled circuit for Eq. (2) (but omitting the square root because it does not affect the ordering) with $M=500$ and $\lambda=128$. With 8-bit RSS values, $s_{m}-\hat{s}_{m}$ requires $256 \mathrm{~B}$. Squaring the result requires $3840 \mathrm{~B}$. To simplify, we assume that accumulating the 500 squares (16-bit values) is done with 25 -bit additions ${ }^{1}$ (each $800 \mathrm{~B}$ ) and, then, we get that the total circuit becomes about $2.33 \mathrm{MB}$. The corresponding numbers with 2-bit RSS values are $64 \mathrm{~B}$ (2-bit addition), $192 \mathrm{~B}$ (2-bit multiplication), 416 B (13-bit addition), and 0.32 MB.

Above, we considered only computing a single instance of Eq. (2) but, in reality, we need to compute several distances and, then, find the shortest of them, e.g., as shown in [23].

\section{B. Paillier Encryption}

In the following, we use Paillier's additively homomorphic public key encryption scheme [24] as an example of how reducing the precision of RSS values can significantly reduce the number of ciphertexts that needs to be communicated. We discuss Paillier encryption because it is used in [8] for privacypreserving localization, but similar advantages can be achieved for most additively homomorphic encryption schemes.

In Paillier encryption, the encryption and decryption functions with a key pair $(s k, p k)$ are as follows:

$$
\begin{aligned}
c & =\operatorname{Enc}(p k, m) \\
m & =\operatorname{Dec}(s k, c)
\end{aligned}
$$

where $m \in \mathbb{Z}_{n}=\{0,1,2, \ldots, n-1\}$, where $n$ is a large integer (more than 2000 bit), $m$ is the plaintext message, $c$ is the ciphertext, $s k$ is the secret key, and $p k$ is the public key. Paillier encryption is additively homomorphic and, therefore, given two ciphertexts $c_{1}$ and $c_{2}$, which are encryptions of $m_{1}$ and $m_{2}$, there is an operator $\star$ for the ciphertexts such that $c_{3}=c_{1} \star c_{2}$ and $\operatorname{Dec}\left(s k, c_{3}\right)=m_{1}+m_{2}$. For Paillier, $\star$ is multiplication modulo $n^{2}$. This allows the server to compute Euclidean distances using the user's encrypted RSS measurements without learning their real values [3], [8].

As shown above, Paillier encryption allows encrypting very large numbers because $n$ is large. Hence, it is not immediately obvious how reducing the precision of RSS values plays any role. However, as shown in [25], it is possible to reduce the number of ciphertexts sent from the server to the user by packing several $b$-bit Euclidean distances into one ciphertext. The packing can be done by, first, scaling the ciphertext of $d_{i}$ by $2^{(i-1) b}$ via repeated homomorphic additions with itself and, second, by adding several scaled ciphertexts together homomorphically. Fig. 2(a) shows how most of the $\log _{2}(n)$-bit plaintext space of a Paillier ciphertext is wasted if only one $b$-bit

\footnotetext{
${ }^{1}$ In practice, the first additions can use a smaller precision
}

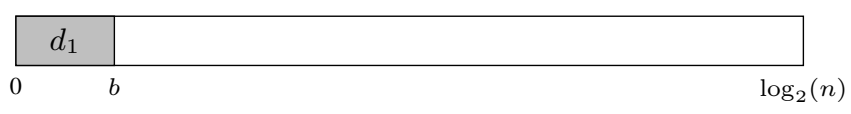

(a)

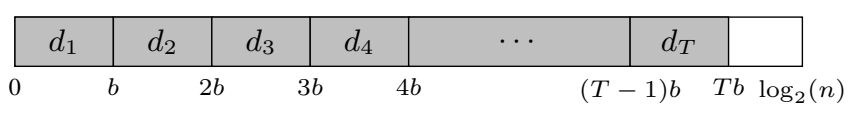

(b)

Fig. 2. (a) Only one $b$-bit distance per ciphertext that could store a $\log _{2}(n)$-bit plaintext (b) $T$ distances packed in one ciphertext for efficient use of the plaintext space

distance is stored in a ciphertext. Fig. 2(b) demonstrates how $T=\left\lfloor\log _{2}(n) / b\right\rfloor$ distances can be packed in one ciphertext for efficient use of the plaintext space. Obviously, the number of distances that fit into a ciphertext depends on $b$, the number of bits per distance, which in turn depends on the RSS values.

Example: Let $\log _{2}(n)=2048$ and assume that Euclidean distances are computed with Eq. (2) by omitting the square root (not possible with Paillier but also no effect on the ordering). Let the number of ANs be $M=500$ and the number of reference points be $N=1000$. If only one distance is stored in one ciphertext, then 2048000 bit need to be transmitted regardless of $b$. With 8-bit RSS values, we have that each $\left(s_{m}-\hat{s}_{m}\right)^{2}$ can be a 16-bit value and as we have $M=$ 500 , one distance can be at maximum a 25-bit value. Hence, $T=\lfloor 2048 / 25\rfloor=81$ values can be packed in one ciphertext resulting in $\lceil 1000 / 81\rceil \cdot 2048=26624$ bit to be transmitted. With 2-bit RSS values, each distance is only a 13-bit value and $T=157$ which gives that only 14336 bit need to be transmitted leading to a $46.5 \%$ saving compared to 8-bit values (and $99.3 \%$ compared to the non-packed version). This clearly shows that the precision plays an important role.

\section{RSS QUANTIZATION METHODS}

This section details the fix bit-length quantization of RSS for secure multi-party computation in support of privacy preserving localization systems.

A quantizer is specified by a codebook and a partition. The codebook defines a finite set $\left\{y_{1}, y_{2}, \ldots, y_{N}\right\}$, the possible $N$ output levels, and the $N$ partition cells that form the input range of the quantizer. The partition cells are specified by their endpoints $\left\{x_{1}, x_{2}, \ldots, x_{N}\right\}$, also called boundary points or decision levels. Quantizers are typically regular, that is, each partition cell is an interval of the corresponding boundary points $\left(x_{i-1}, x_{i}\right)$ and $y_{i} \in\left(x_{i-1}, x_{i}\right)$.

The overall goal when designing a quantizer is to minimize the (squared) error that the quantization $y=Q(x)$ introduces: $d=|x-y|^{2}$ [26]. The performance of a quantizer depends of course on the quantizer itself, but also on the data, $X$, which we model as random variable with a probability density function (pdf) $f_{X}(x)$. A more general and informative measure of a quantizer's overall performance is then the average distortion $D=\mathbb{E}[d(X, Q(X)][26]$, where $\mathbb{E}$ denotes the expectation. To find an optimal quantizer, the codebook and the partitions need to be found at the same time. 


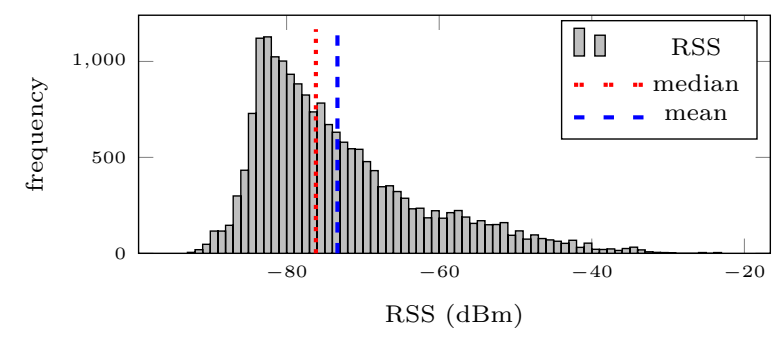

Fig. 3. Histogram of RSS of fingerprint database.

The RSS in a fingerprint database are spatio-temporal samples of an underlying random process whose analytic model is unknown. We design therefore the quantizer based on the empirical distribution of a RSS data set, considering all RSS in a fingerprint database. Fig. 3 illustrates the histogram of the RSS for a three-floor university building. We consider two principle options in this study: uniform quantization and non-uniform quantization. The motivation of studying also the non-uniform quantizers comes from the fact that the RSS probability distribution is not uniform, as shown in Fig. 3.

\section{A. Uniform quantizers and non-uniform quantizers}

Uniform quantizers are characterized by an input-output function that lies on a line with unit slope. That implies equally spaced boundary points and also output levels, $\Delta=y_{i}-y_{i-1}$. Thus, the output levels are given as the midpoints of the quantization intervals, $y_{i}=\left(x_{i}+x_{i-1}\right) / 2$ [26]. This limits the maximum quantization error to $\Delta / 2$ regardless the distribution of the input data.

Non-uniform quantizers adapted to the input pdf may show a smaller distortion than uniform quantizer. Non-uniform quantizer adapt to the pdf, meaning smaller quantization levels for frequent values and more coarse quantization for less frequent values. This results in smaller errors for frequent input values, which may compensate the larger errors yielded from less frequent values and thus decreases the distortion in comparison with uniform quantizer. This enables higher dynamic ranges without an increase of the distortion.

\section{B. Codebook and partition choice}

In order design a quantizer, the optimal partitions for a given codebook must be found. We first compute the codebook based on the complete set of RSS of a fingerprint database and then we fix the partition. The number of bits determines the number of output levels $N=2^{\ell}$.

The codebook of the uniform quantizer is simply determined by picking $N$ equally spaced values from the interval defined by the maximum and minimum of the RSSs: $y_{i}=y_{i-1}+\Delta$, where $y_{1}=\min X$ and $\Delta=(\max X-\min X) /(N-1)$.

The codebook of the non-uniform quantizer is determined in two steps: First, we determine a vector of $N$ equally spaced ordinal numbers starting at one and ending at the cardinality of the RSSs fingerprint data set, $\mathbf{v}=(1,[|X| /(N-1)], \ldots,|X|)$. The $[\cdot]$ is the rounding operator and $|\cdot|$ is the cardinality. Second,

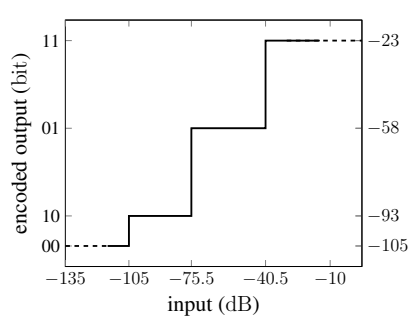

(a) uniform quantizer

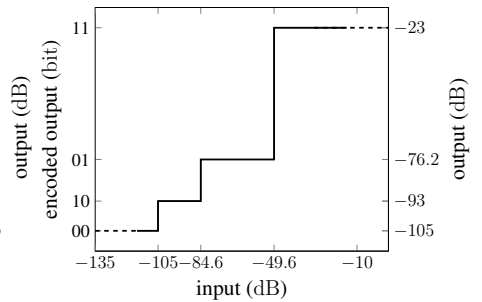

(b) nonuniform quantizer
Fig. 4. Uniform and non-uniform quantizer with zero-bit for the same RSS data set. The threshold for the zero-bit is $s_{\text {th }}=-105 \mathrm{dBm}$.

we rank the set of RSSs. The codebook is then the ordered set of RSS that corresponds to the ranks contained in $\mathbf{v}$.

An optimal partition for a given codebook should minimize the distortion. Thus, the input values in the range of the partition cell $i$ should be closer to $y_{i}$ than to any other output level. This is equivalent to choosing the partition boundary points as midpoints of the neighbouring output levels $x_{i-1}=\left(y_{i-1}-\right.$ $\left.y_{i}\right) / 2$, which is known as the nearest neighbour condition [26]. We chose the partition of the uniform and the non-uniform quantizer according to that rule.

In a last step we encode the quantizer outputs with a simple binary code. Here, the binary number does not have to reflect the actual RSS value as long the same encoding is used on the server and user side. Because in RSS fingerprinting only the difference between the RSS matters (see the metrics in Sect. II).

\section{Modified (combined) quantizer}

Furthermore, we introduce here also a modification of the two quantizers described in the Sect. IV-A. The modified quantizers reserve an extra bit for the ANs whose signals could not be received. This information is either directly or indirectly contained in a fingerprint database: indirectly, if the identifiers of certain ANs do not appear in a fingerprint but in other fingerprints; directly, if the RSS values of every AN are included in the database but are set to some invalid value.

We compute the codebook of these modified quantizers as described before, but with a number of output levels $N=2^{\ell}-1$. Based on these codebooks, we determine the partition, also as described before, and then add an extra output level and boundary point to accommodate the retained zero-bit. We use a value below the lowest RSS of $R S S_{\mathrm{th}}=-105 \mathrm{~dB}$ for the not heard RSS. This zero-bit makes the uniform quantizer clearly nonuniform. Nonetheless, we use the term "uniform" to refer to the modified quantizer derived from the uniform quantizer. The resulting uniform and non-uniform quantizers for the same data set are depicted in Fig. 4(a) and (b).

\section{Positioning RESUlts With QUANTIZATION}

This section presents the experimental set-up and fingerprinting positioning results with quantized RSS. We first compare the positioning accuracy and floor detection rate (FDR) for the uniform quantizer, with and without the zero-bit for not received signals. Then, we present positioning results for the uniform and the nonuniform quantizer of five different RSS data sets. 
TABLE I

CHARACTERISTICS OF FINGERPRINT DATABASES USED IN THE EXPERIMENTS. THE BASE AREA IS ROUGHLY ESTIMATED FROM THE POSITIONS OF THE FINGERPRINTS.

\begin{tabular}{lcccc}
\hline & base area $\left(\mathrm{m}^{2}\right)$ & \# AN & \#FP & \# floors \\
\hline Data-set-1 & $176 \times 73$ & 509 & 628 & 4 \\
Data-set-2 & $176 \times 73$ & 331 & 360 & 5 \\
Data-set-3 [27] & $166 \times 199$ & 489 & 446 & 3 \\
Data-set-4 & $183 \times 163$ & 653 & 406 & 3 \\
Data-set-5 [28] & $395 \times 275$ & 465 & 19861 & 5 \\
\hline
\end{tabular}

\section{A. Measurement environments}

To evaluate the positioning with quantized RSS we use fingerprinting with WLAN RSS. Nonetheless, we expect similar results for RSS-based fingerprinting methods in other networks, such as Bluetooth. We use RSS data from five different data sets, collected in different buildings, with different devices. Details about the different environments can be found in Tab. I. Among the buildings there are three typical university buildings with primarily office and lab use (Data-set-1 to Data-set-3), but also a shopping mall (Data-set-4). Data-set-5 consists of three university buildings. Data-set-1 and Data-set-2 were collected in the same building, but with different devices. Prior to the positioning experiments we determined a quantizer for each data set according to the procedure in Sect. IV.

\section{B. Positioning accuracy with quantized RSS values}

Fig. 5 shows the localization performance of Data-set-3 with uniformly quantized RSS for different bit sizes. In that experiment, we study the influence of the zero-bit and compare the results of the $\mathrm{k}-\mathrm{Nearest}$ Neighbour method (with $k=3$ ) for the three metrics given in the Eqs. (1)-(3).

Noticeable is first of all the high RMSE of the $k$-NN with Euclidean and Sørensen distance if the zero-bit was not used. Interestingly, the Gaussian distance does not show that behaviour. For bit lengths larger than four, the difference between the two quantizer versions is almost negligible. Regarding the FDR, with 1-bit quantization, the methods that use the modified quantizer, with the zero-bit, perform better than the methods without it; and vice versa for bit lengths larger than two.

A comparison of the three distance metrics shows that the Gaussian metric outperforms the other metrics if the RSS are quantized with 1-bit. If two or more bits are spent, the Sørensen distance performs better than the Gaussian distance. The Euclidean distance yields consistently the highest RMSE and lowest FDR.

For the comparative analysis of the uniform and the nonuniform quantizers we refer to Fig. 6. Both quantizers used the zero-bit and the positions were estimated for all five data sets, with the $k$-NN employing the Sørensen distance. This figure depicts the RMSE for different bit lengths and contains the results for the uniform quantizer on the left hand side and the ones for the nonuniform quantizer on the right hand side. Moreover, we estimated the positions with non-quantized RSS and included the RMSE in the graphs.

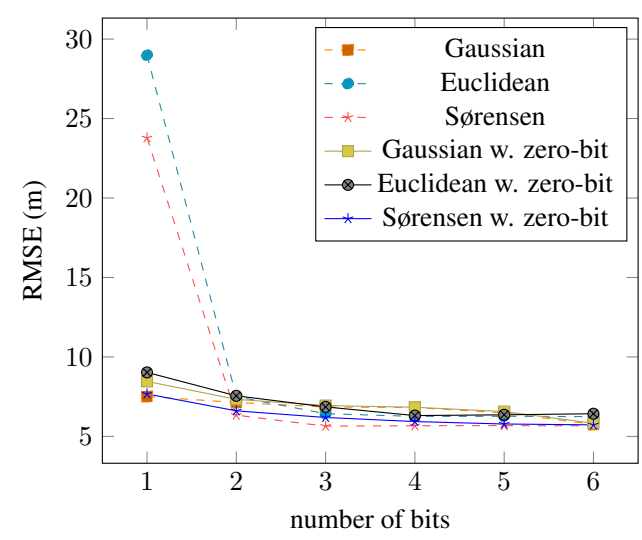

(a)

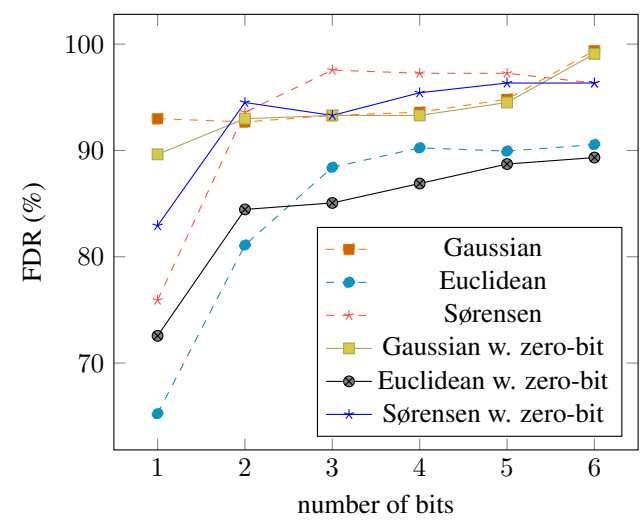

(b)

Fig. 5. Positioning performance of $k$-NN with Gaussian kernel-, Euclideanand Sørensen distance with RSS. The first plot depicts the RMSE and the second plot the FDR.

The uniformly quantized RSS lead to a lower positioning error than the non-uniformly quantized RSS. When a 4-bit quantizer is used, the positioning accuracy is almost as accurate as if the RSS had not been quantized. The accuracy yielded with raw, non-quantized RSS is achieved if we quantize the RSS with 6-bit. That means for WLAN, where RSS are quantized with eight bit, that two bits can be saved in any case without compromising the localization performance. We would also like to point out that for certain applications the accuracy of proximity based positioning (1-bit quantized RSS) might actually suffice. For such applications the storage and computational costs would be decreased significantly.

A final remark is on the positioning accuracy of Data-set-4, whose fingerprints were collected sparsely in an environment that consists of only a few separations: An accuracy of $15 \mathrm{~m}$ is too high for a practical indoor localization system. However, despite the accuracy deviation also this data set conforms with the general pattern regarding the effect of quantization.

\section{CONCLUSIONS AND FURTHER STUDIES}

Through the design of different quantization schemes and the subsequent use of quantized RSS in the positioning methods, we found that uniformly quantized RSSs result in a higher positioning accuracy than using nonuniform quantization; and, 

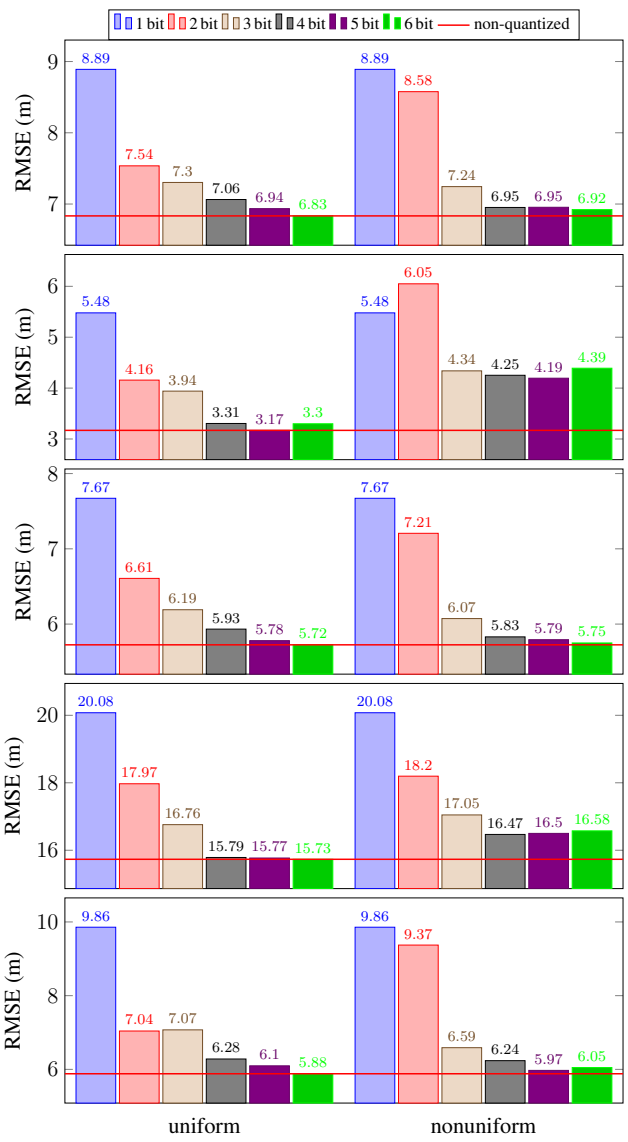

Fig. 6. Positioning performance of $k$-NN with Sørensen distance with RSS for two different quantizers and multiple RSS fingerprint data sets. The data sets from top to bottom correspond the order in Tab. I.

that the zero-bit is only beneficial for localization with binary quantized RSS. Otherwise, we recommend to use all bits in the regular quantizer design. We would like to point out that even 1-bit quantization yields accuracies that might suffice for some scenarios and we consider a $\ell=4$ bit quantization as a affordable w.r.t. positioning performance. Although the Gaussian distance proved to be robust for proximity based positioning, the Sørensen distance yielded the best overall performance.

\section{ACKNOWLEDGMENT}

The authors express their warm thanks to the Academy of Finland (project 303576) for its financial support for this research work. This work has been co-funded by the DFG as part of project E4 within CROSSING and by the BMBF and the HMWK within CRISP.

\section{REFERENCES}

[1] Z. Li, P.-J. Chung, and B. Mulgrew, "Distributed target localization using quantized received signal strength," Signal Process., vol. 134, no. C, pp. 214-223, May 2017. [Online]. Available: https: //doi.org/10.1016/j.sigpro.2016.12.003

[2] E. Lohan, P. Richter, V. Lucas-Sabola, J. Lopez-Salcedo, G. SecoGranados, H. Leppkoski, and E. S. Santiago, "Location privacy challenges and solutions part 2: Hybrid and non-GNSS localization," Inside GNSS magazine, Nov/Dec 2017.
[3] H. Li, L. Sun, H. Zhu, X. Lu, and X. Cheng, "Achieving privacy preservation in WiFi fingerprint-based localization," in INFOCOM 2014, Apr. 2014, pp. 2337-2345.

[4] T. Shu, Y. Chen, J. Yang, and A. Williams, "Multi-lateral privacypreserving localization in pervasive environments," in INFOCOM 2014, 2014.

[5] J. H. Ziegeldorf, N. Viol, M. Henze, and K. Wehrle, "Poster: Privacypreserving indoor localization," WiSec, 2014.

[6] A. Konstantinidis, G. Chatzimilioudis, D. Zeinalipour-Yazti, P. Mpeis, N. Pelekis, and Y. Theodoridis, "Privacy-preserving indoor localization on smartphones," IEEE Transactions on Knowledge and Data Engineering, vol. 27, no. 11, pp. 3042-3055, 2015.

[7] T. Zhang, S. S. M. Chow, Z. Zhou, and M. Li, "Privacy-preserving Wi-Fi fingerprinting indoor localization," in IWSEC, 2016.

[8] Z. Yang and K. Järvinen, "The death and rebirth of privacy-reserving Wifi fingerprint localization with Paillier encryption," in INFOCOM, 2018, https://eprint.iacr.org/2018/259, accessed Mar. 20, 2018.

[9] O. Ozdemir, R. Niu, and P. K. Varshney, "Channel aware target localization with quantized data in wireless sensor networks," IEEE Transactions on Signal Processing, vol. 57, no. 3, pp. 1190-1202, Mar 2009.

[10] H. Shi, X. Li, Y. Shang, and D. Ma, "Cramer-rao bound analysis of quantized RSSI based localization in wireless sensor networks," in International Conference on Parallel and Distributed Systems (ICPADS), vol. 2, 2005, pp. 32-36.

[11] X. Li, H. Shi, and Y. Shang, "A sorted RSSI quantization based algorithm for sensor network localization," in International Conference on Parallel and Distributed Systems (ICPADS), vol. 1, 2005, pp. 557-563.

[12] M. Mizmizi and L. Reggiani, "Design of RSSI based fingerprinting with reduced quantization measures," in International Conference on Indoor Positioning and Indoor Navigation (IPIN), Oct. 2016.

[13] N. Patwari and A. O. Hero, III, "Using proximity and quantized RSS for sensor localization in wireless networks," in ACM International Conference on Wireless Sensor Networks and Applications (WSNA). ACM, 2003, pp. 20-29. [Online]. Available: http: //doi.acm.org/10.1145/941350.941354

[14] R. Niu and P. K. Varshney, "Target location estimation in sensor networks with quantized data," IEEE Transactions on Signal Processing, vol. 54 no. 12 , pp. $4519-4528$, Dec. 2006.

[15] W. Gao, I. Nikolaidis, and J. J. Harms, "RSSI quantization for indoor localization services," in 2017 IEEE 28th Annual International Symposium on Personal, Indoor, and Mobile Radio Communications (PIMRC), Oct. 2017, pp. 1-7.

[16] M. Passafiume, S. Maddio, and A. Cidronali, "An improved approach for RSSI-based only calibration-free real-time indoor localization on IEEE 802.11 and 802.15.4 wireless networks," Sensors, vol. 17, no. 4, 2017.

[17] S. Yiu, M. Dashti, H. Claussen, and F. Perez-Cruz, "Wireless RSS fingerprinting localization," Signal Processing, vol. 131, pp. 235-244, 2017.

[18] P. Davidson and R. Piché, "A survey of selected indoor positioning methods for smartphones," IEEE Communications Surveys Tutorials, vol. 19, no. 2, pp. 1347-1370, Secondquarter 2017.

[19] A. C.-C. Yao, "How to generate and exchange secrets," in FOCS 1986. IEEE, 1986, pp. 162-167.

[20] S. Zahur, M. Rosulek, and D. Evans, "Two halves makes a whole - reducing data transfer in garbled circuits using half gates," in EUROCRYPT, ser. LNCS, vol. 9057. Springer, 2015, pp. 220-250.

[21] V. Kolesnikov and T. Schneider, "Improved garbled circuit: Free XOR gates and applications," in ICALP, ser. LNCS, vol. 5126. Springer, 2008, pp. 486-498.

[22] T. Schneider, Engineering Secure Two-Party Computation Protocols: Design, Optimization, and Applications of Efficient Secure Function Evaluation. Springer, 2012.

[23] E. M. Songhori, S. U. Hussain, A.-R. Sadeghi, and F. Koushanfar, "Compacting privacy-preserving $k$-nearest neighbor search using logic synthesis," in DAC. ACM, 2015, pp. 36:1-36:6.

[24] P. Paillier, "Public-key cryptosystems based on composite degree residuosity classes," in EUROCRYPT, ser. LNCS, vol. 1592. Springer, 1999, pp. 223-238.

[25] A.-R. Sadeghi, T. Schneider, and I. Wehrenberg, "Efficient privacypreserving face recognition," in ICISC, ser. LNCS, vol. 5984. Springer, 2009, pp. 229-244.

[26] A. Gersho and R. M. Gray, Vector quantization and signal compression. Kluwer Academic Publishers, 1992. 
[27] P. Richter, E. S. Lohan, and J. Talvitie, "WLAN (WiFi) RSS database for fingerprinting positioning," Jan. 2018. [Online]. Available: https://doi.org/10.5281/zenodo.1161525

[28] J. Torres-Sospedra, R. Montoliu, A. Martnez-Us, J. P. Avariento, T. J.
Arnau, M. Benedito-Bordonau, and J. Huerta, "Ujiindoorloc: A new multi-building and multi-floor database for WLAN fingerprint-based indoor localization problems," in International Conference on Indoor Positioning and Indoor Navigation (IPIN), Oct. 2014, pp. 261-270. 